\title{
ARQUEOLOGÍA POLICROMA. EL USO Y LA ELECCIÓN DEL COLOR EN EXPRESIONES PLÁSTICAS
}

\section{POLYCHROME ARCHAEOLOGY. COLOR USE AND CHOICE IN PLASTIC ART EXPRESSIONS}

\section{FLORENCIA ÁVILA*}

\begin{abstract}
El color es una de las vías mediante la cual el mundo (cosas, personas, lugares) se incorpora sensiblemente a la práctica social. Actualmente, los modos de abordar la variación cromática desde la arqueología se han basado en mediciones con la Carta Munsell de colores tierra, o análisis físicos y químicos de pigmentos. Pero si nuestro objetivo es reincorporar el color a la experiencia de las personas, tendremos que analizarlo con relación al lugar que ocupa en situaciones reales. Por ello trabajaremos a partir de los fenómenos perceptivos que produce la relación entre colores, proponiendo así nuevas herramientas metodológicas para el tratamiento de materiales arqueológicos.

Palabras clave: color, cerámica arqueológica, fenómenos perceptivos
\end{abstract}

Color is one of the ways in which the world (people, places, things) is consciously incorporated into social practice. Currently, color variation is most commonly treated in archaeology on the basis of Munsell Color Chart measurements or physical and chemical analyses of pigments. However, if our goal is to reincorporate color into buman experience, then we must analyze it in connection with the role it plays in real-life situations. We will therefore examine the perceptual phenomena produced by relations among colors and use these to propose new methodological tools for analyzing archaeological materials.

Key words: color, archaeological ceramics, perceptual phenomena
Nuestra propuesta en este trabajo es centrar la atención en el color de los objetos en el pasado. ¿Por qué? Porque el color es una de las vías fundamentales mediante las cuales el mundo (las cosas, las personas) se incorpora "sensiblemente" a la experiencia y, a través de ella, a la práctica social. Bajo esta premisa, es importante tener en cuenta los fenómenos perceptivos que unen los objetos coloreados a los actores. Considerar algunos de estos aspectos sensitivos nos permitirá poner de relieve la experiencia del color.

Vivimos en un mundo con elecciones cromáticas permanentes. Un mundo "coloreado", siendo el color tanto el fondo como el marco de la experiencia vivida. Si todo está imbuido por el color, ¿por qué la elección de algunos es más importante que la de otros? ¿Cómo operan ciertos colores en el desarrollo de las prácticas sociales? ¿Bajo qué criterios se eligen? ¿Qué hace que algunos tengan más "protagonismo" que otros? La creación y el uso de una paleta entraman diversas prácticas que le dan valor a la tonalidad, desde el proceso de extracción y producción (a través de una combinación de minerales, agua y elementos biológicos), pasando por el uso, la circulación y el consumo. De este modo, los colores, a lo largo de su historia de vida, suponen múltiples prácticas y encuentros con lo material ( $m a$ terial engagements), configurando así una red social y de sentido que involucra personas, objetos, acciones, gestos, momentos y lugares.

\footnotetext{
* Florencia Ávila, Conicet-INAPL, 3 de febrero 1378, Buenos Aires, Argentina, email: florenciaavila@gmail.com
} 
Dicho esto, aclaramos que no es nuestra intención disociar el color de la iconografía, el objeto en que se plasma, los materiales con que está hecho o sus usos. Sin embargo, creemos que es importante en una primera instancia distinguir analíticamente estos aspectos de la realidad para así ahondar en las posibilidades que nos ofrece su conocimiento. Por tal razón, en este trabajo nos centramos en discutir herramientas teórico-metodológicas para el estudio de manifestaciones cromáticas, entendiendo que este es solo un primer paso en la comprensión del papel del color en la materialidad de la práctica social. Hacia el final del trabajo, y solo a modo de ejemplo, aplicamos algunas de estas herramientas al estilo alfarero Yavi-Chicha, correspondiente al período prehispánico Tardío (ca. 1000-1550 DC) en los valles meridionales de Bolivia.

\section{EL ESTUDIO DEL COLOR}

Los fenómenos cromáticos han sido objeto de investigación en distintas disciplinas. Partiendo de que todos los seres humanos tienen capacidad de discriminar colores, se puede afirmar que la percepción del color es una característica universal del aparato cognitivo humano. Desde esta premisa se han desplegado abordajes filosóficos, psicológicos, artísticos, lingüísticos y neurofisiológicos al estudio del color. Los enfoques neurofisiológicos, por ejemplo, se preguntaron si los colores son algo "real", que se encuentra "afuera" en el mundo, o si son producidos por nuestro cerebro como expresiones sensitivas. Cualquiera fuera la alternativa elegida, esta aproximación buscó definir lo más "objetivamente" posible los estímulos cromáticos, dándoles un valor cuantitativo (Hardin \& Maffi 1997; Saunders 2002). Desde un punto de vista antropológico, el problema de estos enfoques radica en que nuestra percepción y cognición se encuentran indefectiblemente influidas por la experiencia (cultural) previa y por el contexto (social) de interacción (Toren 1993). Nuestra experiencia no está solo constreñida por la fisiología de nuestros sentidos, sino también por el sentido que damos a lo percibido, por cómo lo empleamos en el hacer y el relacionarnos (Dedrick 2002).

Otra forma de acercamiento al color fue proponerlo como un sistema de clasificación propio del lenguaje. Esta posición se basa en el supuesto de que la discriminación de la tonalidad está de alguna manera unida a la existencia de términos para los colores, lo que lleva a preguntarse por la naturaleza de la relación entre percepción cromática y lenguaje: ¿podemos conocer colores no categorizados por la lengua? ¿Hay un vínculo directo entre lo que conocemos y lo que podemos decir sobre el color? (Hardin \& Maffi 1997: 355). Berlin y Kay (1996 [1969]) fueron los pioneros en plantear de una manera sistemática esta relación. Tomaron las nociones de colores básicos sugiriendo que su percepción era neurofisiológicamente determinada $\mathrm{y}$, por lo tanto, de naturaleza universal. Al querer estudiar la percepción universal en contraposición con la elección lingüística que determina la percepción de los colores, quisieron identificar los colores básicos o elementales tomando como marco de referencia la Carta Munsell. Definieron estos colores elementales por su generalidad -en tanto sus significados no estuvieran subsumidos en otro término (v. gr., verde manzana, azul petróleo) y fueran aplicables a una gran diversidad de objetos- y por su saliencia, una gran frecuencia y consenso en su uso dentro de una misma lengua. A partir de esto propusieron la existencia de distintos estadios culturales que dividirían los colores desde su percepción, de lo más simple a lo más complejo. El nivel más básico distinguiría dos colores: blanco y negro. En el segundo nivel aparecería la tríada blanco, negro y rojo. Ya en un escalón superior se agregaría a los tres anteriores el verde o el amarillo, para luego seguir complejizándose llegando al círculo cromático. Este modelo ha sido severamente criticado por múltiples autores principalmente por su premisa de que "el lenguaje determina la percepción" (Wierzbicka 1990; Lucy 1997; Saunders \& van Brakel 1998; Jones \& MacGregor 2002, entre otros).

En la antropología simbólica, en cambio, el énfasis estuvo puesto en la búsqueda de reglas universales que dieran cuenta del significado de tonalidades específicas, particularmente en la tríada del negro, rojo y blanco, especialmente en el contexto de performances rituales (Barth 1975; Turner 1980). Aunque estos trabajos muestran que los colores poseen significados que representan cierto conocimiento compartido (Munn 1973) o lo comunican (Morphy 1991), no permiten concluir que estas sean las únicas cosas que los colores hacen ni las más relevantes en el seno de una cultura determinada. Además, al focalizarse solo en los momentos rituales y los objetos de arte, estos estudios dejaron de lado otros aspectos de la vida social, ignorando el papel activo del color en contextos mundanos y en el flujo de la experiencia cotidiana.

Más allá de las críticas mencionadas, todos estos enfoques comparten un problema que es especialmente relevante para la arqueología. Al poner de relieve las respuestas neurológicas a estímulos descontextualizados, su clasificación lingüística, su simbolismo o su función comunicativa generan un alejamiento de la realidad material del color. Al pasar por alto lo que la gente hace con los objetos coloreados $\mathrm{y}$, recursivamente, lo que ellos le hacen a la gente dentro de la dinámica de 
la práctica social, lo "des-materializan" (Owoc 2002; Miller 2005; Young 2006). ¿Cómo reinsertar el color en el mundo material?

\section{EL CONTRASTE COMO HERRAMIENTA PARA EL ABORDAJE DEL COLOR}

Nuestro punto de partida es analizar cómo se confeccionan ciertos conjuntos de objetos en patrones cromáticos reiterados, generando una unidad (perceptiva) que es prácticamente reconocida por un grupo de personas en un tiempo y en un espacio determinado. Buscamos identificar configuraciones particulares que producen efectos que se experimentan de forma compartida, resultando en prácticas comunes.

La Teoría de la Gestalt o Psicología de la Forma es una de las corrientes que más ha estudiado la percepción como parte integral de la experiencia. Este cuerpo teórico ha tenido como principal objetivo abordar el problema de la procedencia del significado en las artes visuales, preguntándose cómo se perciben y qué comunican las mismas. ${ }^{1}$ Con este fin la Gestalt desarrolló un cuerpo de principios a partir de experimentaciones sobre percepción sensorial (principalmente visual), fundamentando que cualquier acontecimiento visual es una forma con contenido, pero el contenido está intensamente influido por la significancia de las partes que lo constituyen, como el color, el tono, la textura, la dimensión, la proporción y sus relaciones compositivas con el significado (Gombrich 1999). De acuerdo con esto, los psicólogos de la Gestalt enumeraron ciertos principios de la forma, también llamados fenómenos perceptivos, como: a) principio de agrupamiento (por proximidad, semejanza y posición); b) principio de buena continuación o mejor dirección (el movimiento que se percibe); c) principio de buena curva; d) principio de la buena forma; e) principio de cerramiento; f) principio de figura y fondo; g) principio de pregnancia; h) principio de transportabilidad (simetrías). Estas operaciones no se autoexcluyen y están directamente relacionadas con cualidades pictóricas tales como la configuración, el tamaño, la posición, la actitud, el valor, el color y la textura (Scott 1950; Wong 1979; Kanizsa 1986).

Como nuestro interés radica en los efectos cromáticos y en los fenómenos perceptivos que ellos disparan, primero tendremos que desmenuzar los componentes del color, para luego jugar con las formas en que se pueden interrelacionar, derivando en efectos particulares. Los componentes cromáticos se definen por tres variables físicas y gráficas. El tinte (bue) es el valor diferencial de un color o su magnitud pregnante, definiéndose en relación con otro color o en relación con su posición situacional frente a los demás (monocromos, análogos, alternos, etc.); es decir, qué color es. ${ }^{2} \mathrm{El}$ valor (value o tone) es el grado de luminosidad que tiene un color, la medida de la cantidad de gris que posee; cuanto más luminoso es un color, su valor o matiz es más alto y viceversa. Por último, la saturación (saturation o chroma) es el grado de pureza o brillantez que posee un color cuando carece de blanco o de negro (Moles \& Janiszewski 1992) (fig. 1).

El color es esencialmente "inestable", no puede definirse si no es con relación a otro (como sucede en el círculo cromático). En este sentido los colores son mutuamente dependientes. La naturaleza relacional del color es particularmente evidente en situaciones de contraste, razón por la cual es una de las resoluciones cromáticas más recurrentemente utilizadas. Proponemos, entonces, centrarnos en los contrastes, aunque aclarando que estos pueden ser rígidos o flexibles, suaves o severos, obvios o difusos, simples o complejos; el contraste
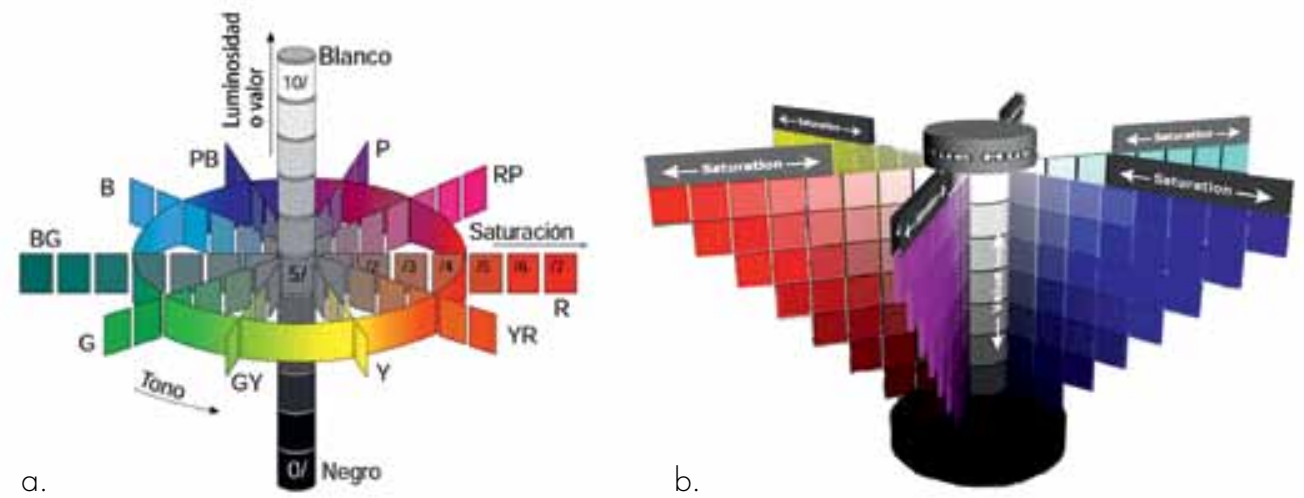

Figura 1. Estructura cromática: a) Interrelación entre tinte, valor y saturación; b) Esquema tridimensional de las variables cromáticas utilizadas en la estructura de la tabla Munsell.

Figure 1. Chromatic structure: a) Interrelation of tint, value and saturation; b) Three-dimensional scheme of chromatic variables used in the Munsell table. 
es solo una clase de comparación. Para aplicarlo como herramienta tomaremos los siete contrastes propuestos por Johannes Itten (1961). ${ }^{3}$ Estos son:

Contraste de tintes. Contraste entre dos colores puros en su máxima saturación. La distancia extrema sería entre los colores primarios amarillo/rojo/azul, aunque cualquier tinte llevado a su máximo nivel de saturación puede participar en un nivel de contraste semejante.

Contraste claro-oscuro. Representa la escala de valores de luz de los colores, siendo el negro y el blanco, obviamente, los dos extremos de contraste. Estos niveles no solo pueden presentarse en intervalos precisos, sino también como transiciones imperceptibles, una suerte de glissando que debe ser considerado para evaluar la intensidad del contraste.

Contraste frío-cálido. Generalmente, dentro del círculo cromático, se reconoce como colores cálidos a los amarillos anaranjados, los naranjas, los rojos anaranjados, los rojos y los rojos violáceos, y como fríos al amarillo verdoso, el verde, el azul verdoso, el azul, el azul violáceo y el violeta. Como sucede en todos los tipos de contrastes, los tintes intermedios entre ellos en el círculo cromático pueden ser fríos o cálidos de acuerdo a si son contrastados con tonos más cálidos o más fríos.

Contraste de complementarios. Son colores que están opuestos diametralmente en el círculo cromático. Son opuestos pero se requieren uno al otro. Hay un solo complementario para un color dado, y esto se define cuando sus pigmentos, al ser mezclados, dan un gris neutro. Los colores complementarios más comunes son: amarillo-violeta, azul-naranja, rojo-verde.

Contraste simultáneo. Implica la ilusión óptica de que a partir de la utilización de un color, el mismo exige simultáneamente su complementario y, si no le es dado, lo produce por sí mismo. Por ejemplo, una tela roja con pequeñas bandas negras ejerce un fuerte contraste de simultaneidad. El fondo rojo hace parecer verdosas las bandas negras, generando una molestia visual que puede ser resuelta bajando la intensidad del negro con gotas de azul o marrón.

Contraste de saturación. En tintes puros se aplican blancos o negros produciendo pérdida de luminosidad y una modificación de los atributos. Esto causa un cambio de saturación, generando un contraste opaco-brillante dentro de, por ejemplo, un mismo tinte.

Contraste de área. Es el contraste cualitativo que concierne a la relación del tamaño del área coloreada. Sería el tamaño o la disposición de la mancha de color en comparación con el otro.

A partir de los contrastes anteriormente señalados se pueden cumplir también los siguientes fenómenos (Moles \& Janiszewski 1992):
Ley de diferencia aumentada: en los contrastes de valor, saturación o tinte, pareciera que las diferencias son más grandes de lo que son.

Inducción complementaria: los grises serán inducidos complementariamente de acuerdo al color que tengan al lado.

Asimilación: cuando los matices contiguos son lo bastante semejantes (colores análogos), o cuando las áreas son lo bastante pequeñas, los colores se aproximan entre sí en vez de marcar un contraste.

De acuerdo con estos fenómenos, podemos decir que el color es dinámico e influye directamente en la percepción de las formas coloreadas, ordenando, jerarquizando, resaltando u ocultando diferencias o similitudes, compensando pesos y neutralizando matices. Estos aspectos perceptuales son relevantes al momento de entender cómo inciden los objetos coloreados en los actores dentro de su experiencia. De hecho, algunos autores han planteado que cuando los colores actúan juntos producen en las personas un efecto de fascinación, atracción y hasta seducción (Coote 1992; Gell 1992, 1998; Gage 1993).

La herramienta paradigmática para medir "objetivamente" el color en arqueología es la Carta Munsell de Suelos. La organización de la misma interrelaciona las variables tinte, valor y saturación de forma tridimensional con el fin de proponer ordenamientos cromáticos (fig. 1). ${ }^{4}$ En nuestra disciplina es usada, por ejemplo, para definir una paleta en materiales "coloreados" a partir de los colores tierra codificados por la carta. Estos datos han sido tomados como una "descripción objetiva" del color de los materiales, o han sido utilizados para abordar preguntas sobre especialización artesanal, estandarización o intercambio. Lo cierto es que al analizar un conjunto alfarero, por ejemplo, la categorización de un color suele estar dada por una gran cantidad de lecturas Munsell. Piezas o conjuntos caracterizados por una tonalidad roja, o por un contraste negro sobre crema, dan una variabilidad de lecturas dentro de cada color que no refleja la percepción que se tiene del conjunto alfarero como unidad. ¿Qué alcance interpretativo tiene el identificar un color como 2.5 YR 5/4?

Las sociedades usan paletas en las que se prefieren ciertos colores o combinaciones de colores sobre otras más allá de las diferencias microtonales. Es este gesto plástico el que crea una experiencia y una práctica distintiva. Por ello, la utilización aislada de la Carta Munsell corre el riesgo de enmascarar en la diversidad de las lecturas -derivada tal vez de las distintas técnicas, materias primas disponibles o mezclas empleadas para preparar las pinturas- la recurrencia de las consecuencias visuales buscadas por los artesanos y reconocidas 
espontáneamente por los miembros del grupo. Es por esto que, aunque la Carta Munsell es útil para registrar inicialmente el color de los artefactos y para comunicarlo entre investigadores, no puede ser la única herramienta descriptiva o analítica.

Es necesario, entonces, desarrollar una metodología que incluya el "efecto cromático". Para ello resulta interesante tomar en cuenta sistemáticamente los fenómenos perceptivos que generan las expresiones plásticas, como los de reversibilidad, contraste, pregnancia, invisibilidad y enmascaramiento, entre otros, analizándolos no como alternativas excluyentes, sino desde su conjunción perceptual. Por cierto, un proyecto como este excede nuestras posibilidades; en el próximo apartado, sin embargo, ejemplificamos las características de un enfoque de este tipo a través del análisis de un caso.

\section{EL CONJUNTO ALFARERO YAVI-CHICHA}

Al conjunto alfarero Yavi-Chicha se le ha estimado una cronología aproximada que va desde el siglo XI al XVI.
Es el estilo alfarero predominante durante esta época en los valles de la cuenca del río Grande de San Juan, una región que comprende actualmente el norte de la Puna jujeña en Argentina y las provincias de Sud Chichas y Nor Chichas en Bolivia (Krapovickas 1987/1988; Raffino 1988; Krapovickas \& Aleksandrowicz 1990; Ángelo 1999; Ávila 2008, 2009) (fig. 2). Su estudio ha revestido una gran importancia para el entendimiento de las relaciones sociales en el ámbito circumpuneño, dado que piezas de dicho estilo se han encontrado en lugares distantes de su región de origen, en contextos de distintos momentos. Para épocas preinkaicas aparece como material foráneo en la Quebrada de Humahuaca (Cremonte 1994; Nielsen 2007), la puna de Casabindo (Mamaní 1998; Albeck 2007), las tierras bajas tarijeñas (Rendón 2008) y los oasis de San Pedro de Atacama (Tarragó 1989; Stovel 2002). En época inkaica su dispersión se amplió hacia el río Loa (Aldunate 1993), Coquimbo (Cantarutti \& Mera 2004), la provincia de Salta (Cremonte \& Williams 2007) y Potosí (Ibarra Grasso 1973).

Las piezas Yavi-Chicha se reconocen principalmente por su color, dado que poseen cuatro variedades

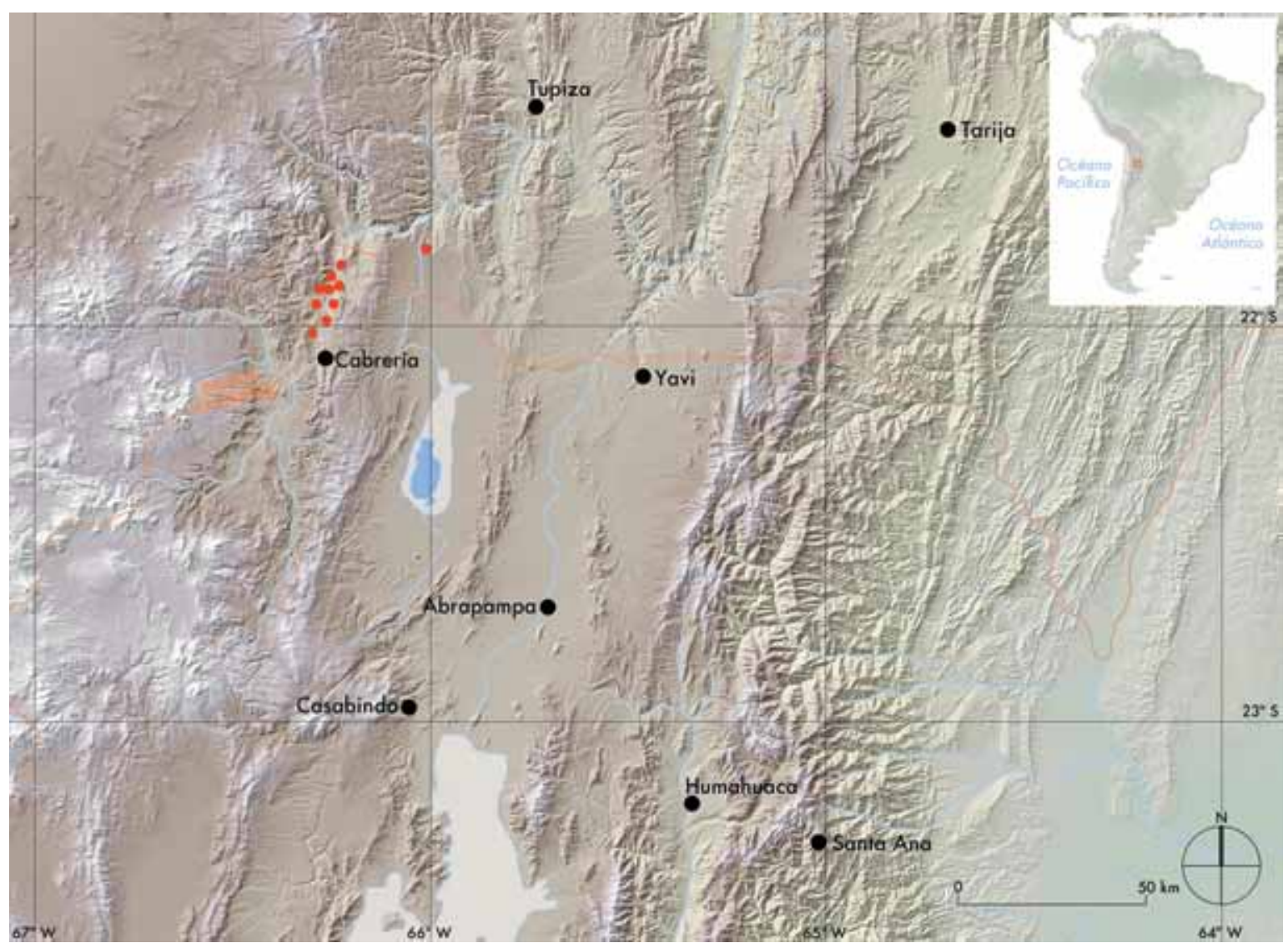

Figura 2. Cuenca del río Grande de San Juan con detalle de la cuenca media especificando en el espacio los sitios trabajados. Figure 2. Basin of the Rio Grande in San Juan, with detail of the middle basin indicating sites investigated. 
cromáticas diferentes que se combinan de forma pautada. Ellas son: morado, ante, rojo y negro desleído; con menor frecuencia se han registrado crema y violáceo. El negro desleído es el único color que se emplea para el diseño pictórico, los demás se utilizan como colores plenos en las piezas, pudiendo ser monocromas (moradas, rojas o antes) o bicromas (moradas sobre ante).

Cuando se emplea la bicromía, se produce un efecto cromático llamativo: la pieza se "divide perceptualmente" en espacios diferenciados, tenga diseño pictórico o no. La experiencia perceptiva del color interviene en el reconocimiento y la delimitación de estas áreas distintas generando una situación de contraste. En términos de Itten (1961) un contraste claro-oscuro dentro de una paleta cálida (morado y ante) que aumentan su diferencia por ser colores alternos; por el contrario, el rojo, su análogo, siempre aparece como único color. El intervalo entre los tintes se mantiene más allá del grado de saturación de los mismos, lo que produce que siempre se llegue a la misma consecuencia cromática. La pregnancia de las piezas está dada por este juego perceptivo, por esta situación de contraste (fig. 3).
Pero en situaciones reales no se interactuaba con solo "una pieza", como hacemos los arqueólogos al analizar los materiales, sino con todo un conjunto de ellas. Esto lleva a pensar que el contraste de claros y oscuros también estaría operando entre vasijas. Los porcentajes de piezas monocromas de los distintos colores son muy similares y se encuentran presentes en todos los contextos, como veremos más adelante. Esto produce que, aunque no estemos a una distancia cercana de los objetos, inmediatamente reconozcamos las combinaciones cromáticas y los colores que las forman, por más variaciones microtonales que cada uno de ellos presente (fig. 4). Si bien estos colores son análogos (ante-rojo-morado) dentro de la gama de los cálidos, la alternancia se refuerza en las piezas bicromas. El contraste claro oscuro sigue operando como el efecto cromático pregnante.

Por otro lado, en los conjuntos expuestos hay una clara diferencia entre piezas "coloreadas" y piezas "no coloreadas" o alisadas, ¿producen un efecto cromático? Aunque las piezas no estén coloreadas, el solo hecho de pulir la pasta o solo alisarla causa un efecto contrastante
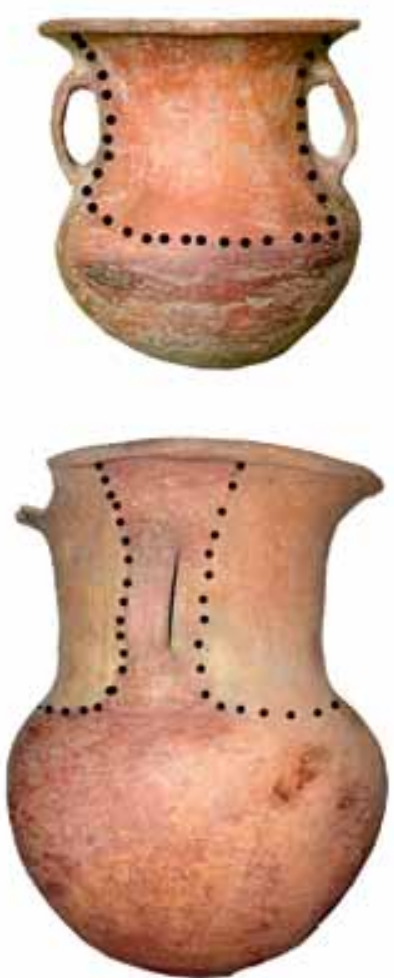
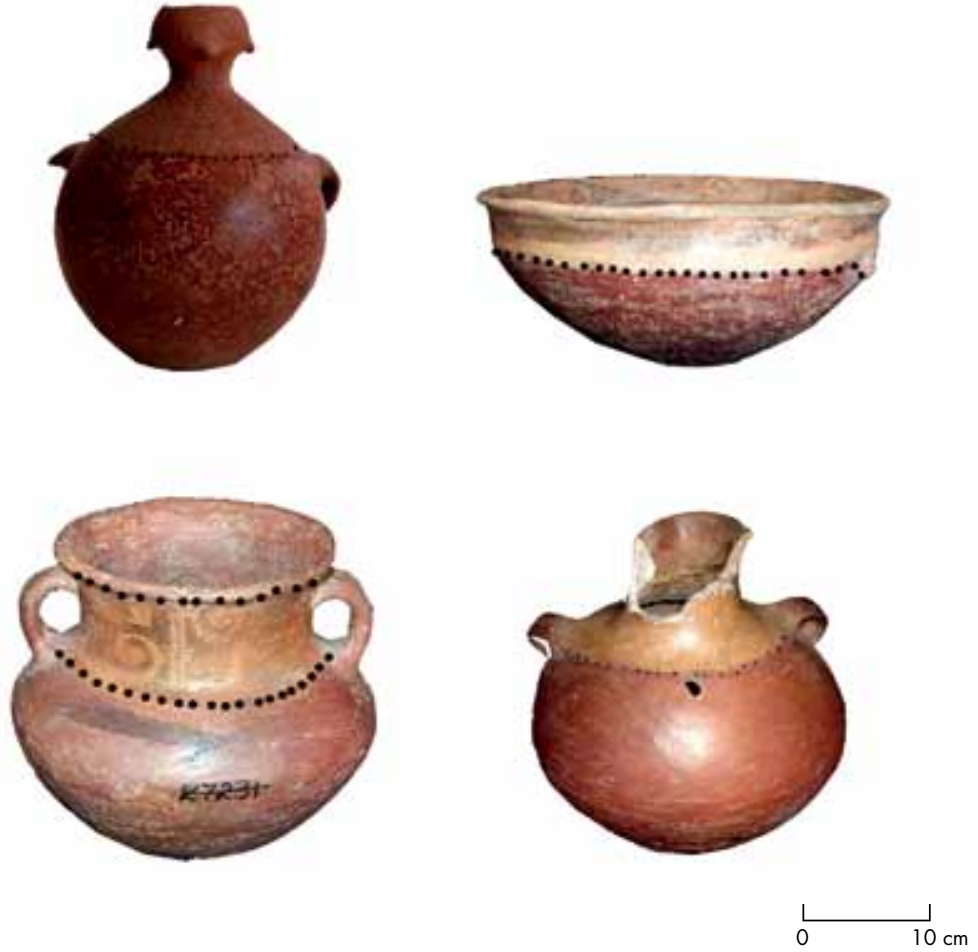

Figura 3. Situación de contraste claro-oscuro del diseño cromático morado sobre ante en piezas enteras bicromas Yavi-Chicha (con línea de puntos la delimitación de los campos de color).

Figure 3. Contrasting dark-light color design (purple over light brown) in whole bichromatic Yavi-Chicha pieces (with dotted lines delimiting color fields). 

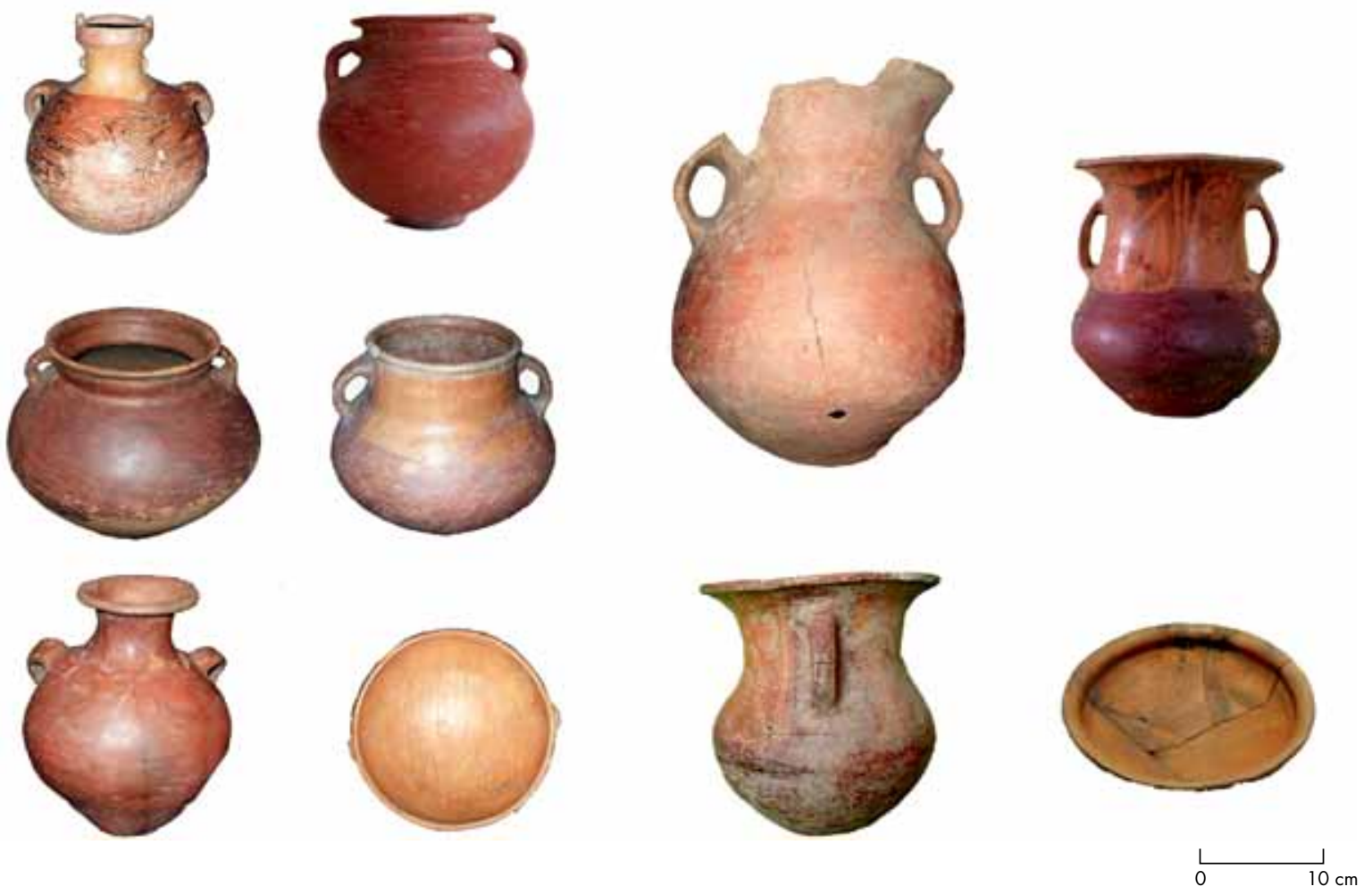

Figura 4. Situación de contraste claro-oscuro entre piezas monocromas y bicromas Yavi-Chicha. Figure 4. Contrasting light-dark designs in monochromatic and bichromatic Yavi-Chicha pieces.

de brillo, es decir, un contraste de saturación. Si a ello le agregamos color a las vasijas y las pulimos, el contraste de saturación se hace aun más evidente ("ley de diferencia aumentada"). Los colores se saturan en brillantez, mientras que los alisados parecen, en relación, sin brillo y opacos (fig. 5).

Estas combinaciones se repiten de forma recurrente en contextos de diferente funcionalidad. Así, se las registra con frecuencias muy similares, tanto en las colecciones de piezas enteras conservadas en museos -en su mayoría procedentes de contextos mortuorios $-^{5}$ como en conjuntos domésticos recuperados mediante recolecciones superficiales y excavaciones estratigráficas en distintos sitios de la cuenca media del río Grande de San Juan (Ávila 2008; Nielsen et al. 2010). ${ }^{6}$ Las recolecciones superficiales se realizaron de forma sistemática abarcando 14 sitios sobre el río Grande de San Juan, desde las localidades de Cabrería hasta el Angosto (Puna de Jujuy, Argentina), y uno en la localidad de Chipihuayco (quebrada de Talina, Bolivia) (ver fig. 2). El material en estratigrafía proviene de una excavación en área en un complejo habitacional y de dos sondeos realizados en basureros, todos del sitio Chipihuayco.

Por ejemplo, si comparamos la cantidad de piezas coloreadas pulidas versus las alisadas observamos las siguientes tendencias. Para la muestra de colecciones $(\mathrm{n}=325)$, el $16 \%$ se encuentra alisado mientras que el $85 \%$, pulido. Suponemos que esto se debe a que su procedencia es mayoritariamente de contextos mortuorios, pero al compararlo con las muestras contextuales las tendencias son muy similares. Tomando solo los fragmentos de borde, de modo de reducir los sesgos en la comparación con piezas enteras, en las recolecciones superficiales $(n=775)$ el $32 \%$ está alisado y el $68 \%$, pulido, mientras que para las excavaciones $(n=338)$ el $41 \%$ está alisado y el 59\% está pulido.

Nos falta un último factor, el diseño pictórico. Este se encuentra subordinado al cromático; el negro desleído se esfuma aun más bajo el contraste de color. Este diseño se plasma de la misma forma en piezas grandes y pequeñas, accesibles y contenedoras, siempre se presenta en tamaño reducido y con tinte desleído. Se produce entonces un contraste de asimilación de un negro aguado sobre un fondo saturado (fig. 6). A diferencia de lo que sucede con los demás colores y sus marcados contrastes, ese profuso diseño, caracterizado por un complejo juego de simetrías y reversibilidades, solo actúa en forma proxémica, seguramente sobre un reducido grupo de actores que pueden observar la vasija a corta distancia o tenerla entre sus manos. Por la baja presencia que 

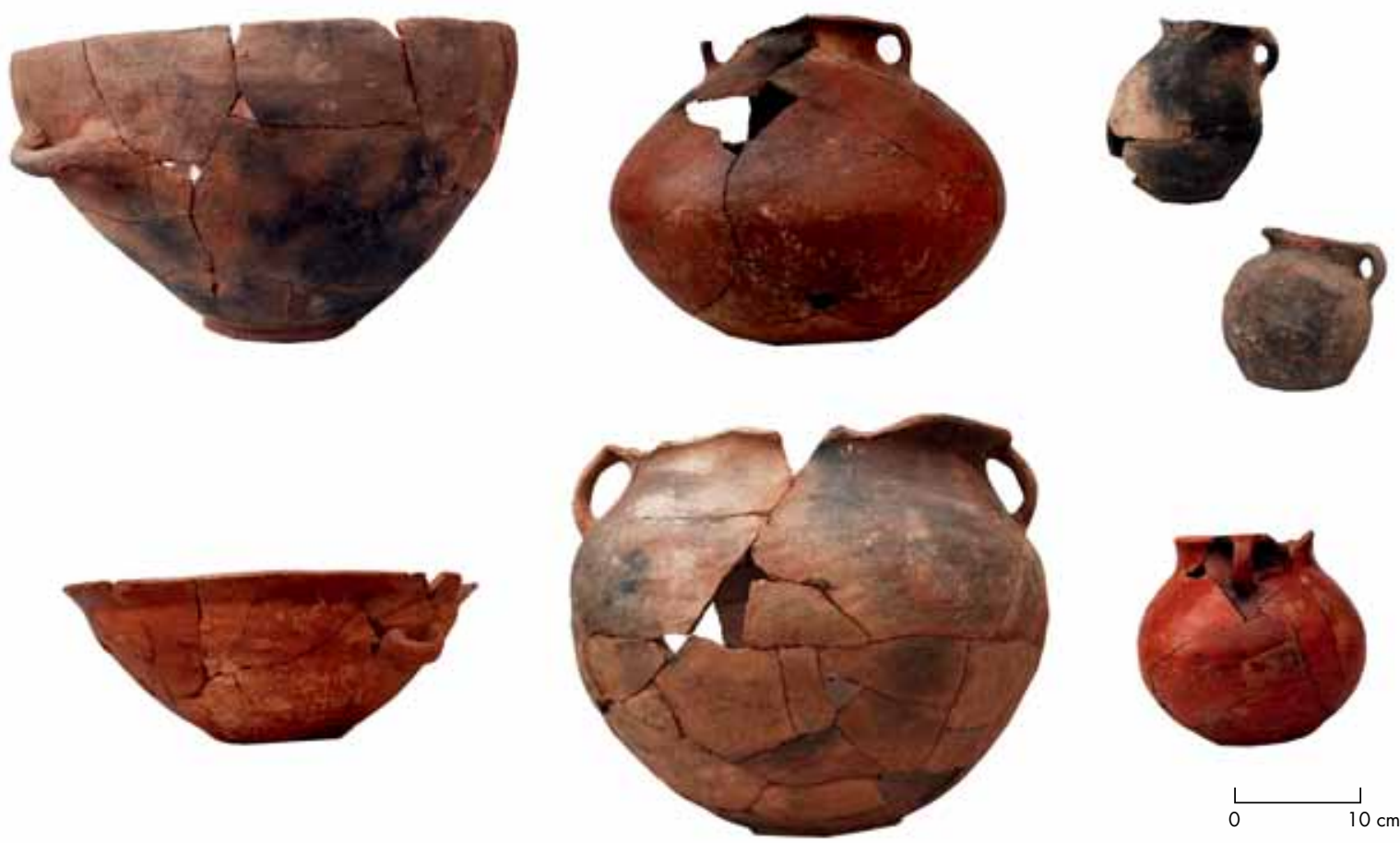

Figura 5. Situación de contraste de saturación entre piezas alisadas y piezas pulidas Yavi-Chicha. Figure 5. Contrasting saturations in smooth and polished Yavi-Chicha pieces.

tenemos en todos los conjuntos estudiados (representan un $48 \%$ para las piezas de colecciones y menos de un $10 \%$ para los fragmentos de los $\mathrm{N}$ anteriormente mencionados) podemos pensar, además, que el contexto de exhibición revestiría situaciones particulares (contextos mortuorios, de circulación, de exposición, etc.).

Estas características del estilo Yavi-Chicha plantean una serie de interrogantes: ¿por qué razón los diseños pictóricos han sido concebidos para ser vistos a corta distancia? ¿Qué prácticas se encuentran implicadas en su uso? ¿Cuál es la relación entre lo casi invisible de lo pictórico y lo evidente del morado, el ante y el rojo en el juego cromático? Estos gestos plásticos parecen estar invitando a distintas prácticas, modos de interacción y formas de entramarnos en la realidad.

La ubicuidad de las vasijas coloreadas, la alta visibilidad de los contrastes de valor que emplean y la reiteración de estas mismas combinatorias en distintos contextos (mortuorios, de actividad doméstica) revela un esfuerzo deliberado por mantener la uniformidad cromática de la experiencia. Esta homogeneidad crearía un sentido del gusto compartido, de afinidad, que reforzaría los vínculos entre los miembros (conocidos o no) de una misma colectividad, distanciándolos sensiblemente de grupos habituados a otros patrones cromáticos.
La escasa visibilidad del diseño pictórico que resulta de su asimilación, en cambio, junto con su complejidad formal, interpelan a un grupo más cercano. En sentido literal, puesto que solo se percibe y aprecia a corta distancia, pero también social, ya que requiere una íntima familiaridad con el repertorio de motivos y configuraciones para valorar la destreza y las sutiles variaciones que conlleva su realización.

\section{CONCLUSIÓN}

En síntesis, abordar el color en forma relacional (Owoc 2002; Young 2006) nos marca algunos caminos potencialmente fructíferos a seguir en su estudio arqueológico. Primero, pone de relieve que las relaciones cromáticas ( $p$. ej., los contrastes) son más relevantes para entender la experiencia cromática que los colores en sí mismos o sus valores absolutos cuantificables a través de dispositivos tales como la Carta Munsell. Sin dejar de lado el uso de métodos "objetivos" o capaces de revelar variaciones microtonales que pueden ser interesantes para abordar preguntas sobre tecnología o producción (entre otras), es importante emplear categorías descriptivas que pongan de relieve los contrastes cromáticos presentes en cada pieza, entre vasijas y, eventualmente, entre la alfarería y 

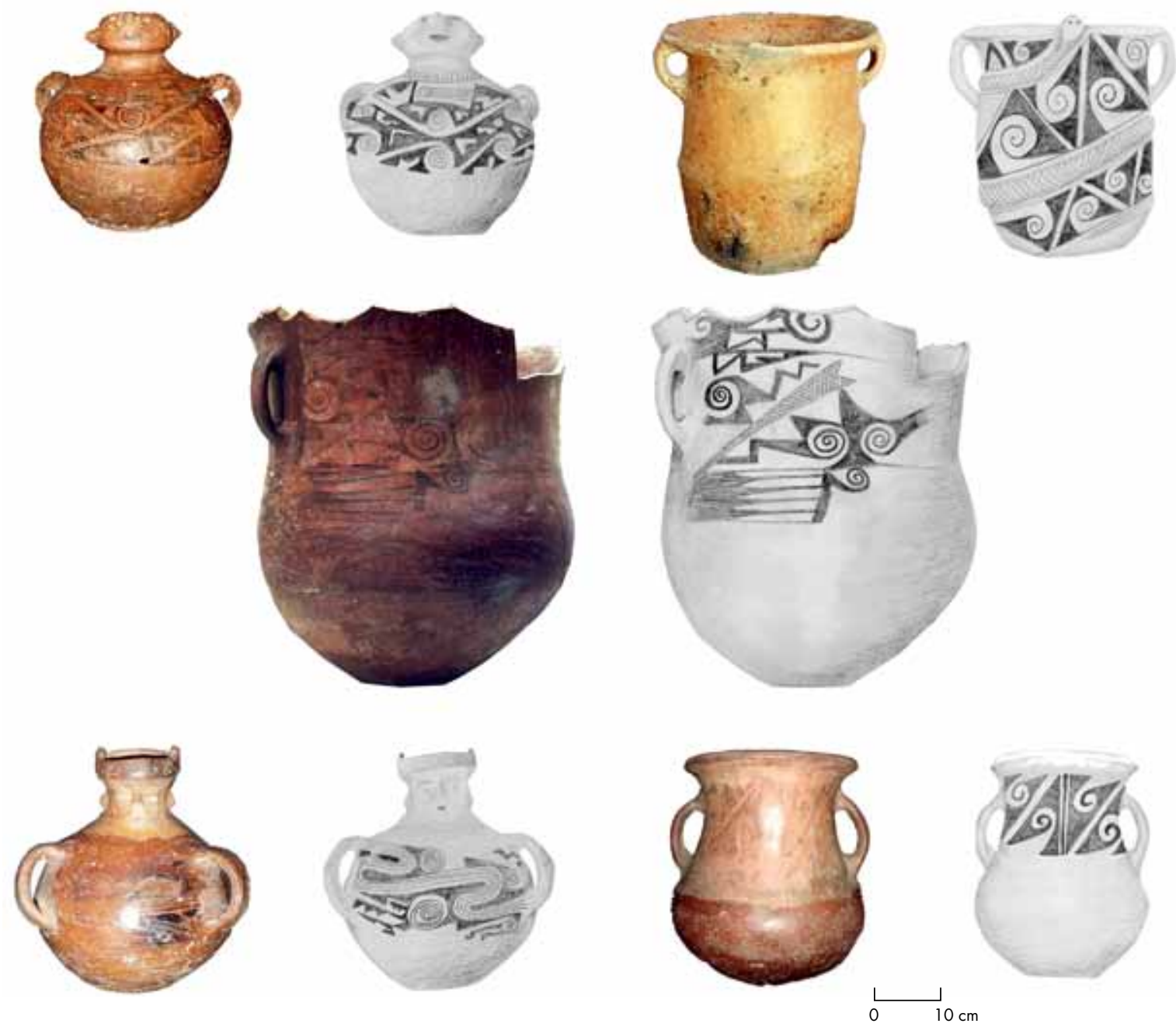

Figura 6. Situación de asimilación del diseño pictórico en piezas Yavi-Chicha. El dibujo resalta la pictografía para apreciar el efecto de asimilación.

Figure 6. Assimilation of pictorial designs in Yavi-Chicha pieces. The drawings highlight the pictographs so the assimilation effect can be appreciated.

otros materiales que integran el contexto, por ejemplo, arquitectura, textiles, entorno físico, etc.

Segundo, nos invita a pensar en los efectos (buscados o no) que estas combinaciones producían en las personas, en sus consecuencias "sociales" (Gell 1998; Latour 2005). ¿Cómo se distribuyen distintos tipos de contrastes en el espacio o en diversos contextos de acción? ¿Qué tan pautadas o regimentadas se encuentran estas relaciones? ¿Qué continuidades o discontinuidades marcan entre las personas entre sus distintas prácticas? Como podemos observar, preguntas que nos llevan, por lo menos para el Estilo Yavi-Chicha, a comenzar a desentrañar el rol de los objetos en el entramado de las relaciones sociales.
RECONOCIMIENTOS Quisiera agradecer los comentarios, las sugerencias y las correcciones tanto de los evaluadores como del comité editorial del Boletín, los cuales me han ayudado a repensar el artículo y poder expresarlo con mayor claridad. Extiendo también mi gratitud hacia mi director, Dr. Axel Emil Nielsen, por el invaluable seguimiento en toda mi investigación y al equipo del PAAS (Malena Vázquez, Juan Maryañski, Julio Ávalos, Karina Menacho) por su generoso trabajo y apoyo. Por último, este artículo e investigación ha sido subvencionado por una beca doctoral del CONicet y un subsidio de la Agencia Nacional de Promoción Científica y Tecnológica (PICT 30059).

\section{NOTAS}

${ }^{1}$ Entre los pioneros en plantear esta problemática podemos mencionar a Christian von Ehrenfels, Max Wertheimer, Wolfgang Köhler, Kurt Koffka, entre otros (Koffka 1973). El investigador que 
le dio mayor alcance, sin embargo, fue Rudolf Arnheim (1983, 1986a, 1986b), quien no se limitó a estudiar el funcionamiento de la percepción, sino que investigó la calidad de las unidades visuales individuales y las estrategias de su unión en un todo final y completo.

${ }^{2}$ Con pregnancia nos referimos a la fuerza de la forma. Es la capacidad de imponerse como imagen mental y en el recuerdo.

${ }^{3}$ Johannes Itten (1961) fue uno de los maestros de la Bauhaus en el desarrollo de los fundamentos y las características de los materiales, la composición y el color. De allí nació la obra The Art of Color, referente hasta hoy en día de los estudios cromáticos.

${ }^{4}$ En la Carta Munsell el eje lineal corresponde al valor y el eje circular corresponde al círculo cromático o tinte. La distancia que hay desde el eje circular hasta el eje de valor nos da el tercer eje que es el de la saturación. Se llega así a una figura de tres dimensiones.

${ }^{5}$ Se analizaron 325 piezas pertenecientes a los museos de Argentina: Eduardo Casanova (Tilcara), Colección Yavi Chico (Yavi Chico), Provincial (Jujuy), Municipal y Mosoj Ñam (La Quiaca), Ambrosetti (Ciudad de Buenos Aires), Ciencias Naturales (La Plata). De Chile: Gustavo Le Paige (San Pedro de Atacama).

${ }^{6}$ La muestra con la que se trabajó fue de 11.427 fragmentos provenientes de 15 sitios de la cuenca del río Grande de San Juan.

\section{REFERENCIAS}

ALBeck, M., 2007. El Intermedio Tardío: Interacciones económicas y políticas en la Puna de Jujuy. En Sociedades precolombinas surandinas: Temporalidad, interacción y dinámica cultural del NOA en el ámbito de los Andes centro-sur. V. Williams, B. Ventura, A. Callegari \& H. Yaccobaccio, Eds., pp. 125-146. Buenos Aires: Universidad de Buenos Aires.

Aldunate, C., 1993. Arqueología en el Pukara de Turi. En Actas del XII Congreso Nacional de Arqueología, Vol. 2, pp. 61-78, Temuco.

Angelo, D., 1999. Tráfico de bienes, minería y aprovechamiento de recursos en la región de los valles del sur boliviano. Tesis de licenciatura en Arqueología, Facultad de Ciencias Sociales, Universidad Mayor de San Andrés, La Paz.

Arnheim, R., 1983. Arte y percepción visual. Madrid: Editorial Alianza forma.

1986a. El pensamiento visual. Barcelona: Editorial Paidós. 1986b. Hacia una psicología del arte. Arte y entropía. Madrid: Editorial Alianza.

Ávila, F., 2008. Un universo de formas, colores y pinturas Caracterización del estilo alfarero Yavi de la Puna nororiental de Jujuy. Intersecciones en Antropología 9: 197-212.

2009. Interactuando desde el estilo. Variaciones en la circulación espacial y temporal del estilo alfarero Yavi. Estudios Atacameños 37: 29-50.

BARTH, F., 1975. Ritual and Knowledge among the Baktamen of Papua New Guinea. New Haven: Yale University Press.

Berlin, B. \& P. KAY, 1996 [1969]. Basic Color Terms: Their Universality and Evolution. Berkeley: University of California Press.

Cantarutti, G. \& R. Mera, 2004. Estadio fiscal de Ovalle: redescubrimiento de un sitio Diaguita-Inca en el Valle del Limarí. Chungara 36: 833-845.

Cооте, J., 1992. Marvels of Everyday Vision: The Anthropology and Aesthetics and the Cattle-Keeping Nilotes. En Anthropology, Art and Aesthetics, J. Coote \& A. Shelton, Eds., pp. 245-273. Oxford: Clarendon Press.

Cremonte, M. B., 1994. Tendencias en relación a la producción y distribución de la cerámica arqueológica de la Quebrada de Humahuaca. En Taller "De Costa a Selva". Producción e intercambio entre los pueblos agroalfareros de los Andes Centro Sur, M. E. Albeck, Ed., pp. 177-198. Tilcara: Instituto Interdisciplinario Tilcara, Universidad de Buenos Aires.

Cremonte, M. B. \& V. Williams, 2007. La construcción social del paisaje durante la dominación Inka en el noroeste argentino. En Procesos sociales prehispánicos en el sur andino. La vivienda, la comunidad y el territorio, A. Nielsen, C. Rivolta, V. Seldes, M. Vázquez \& P. Mercolli, Eds., pp. 207-236. Córdoba: Brujas. Dedrick, D., 2002. The roots/routes of color term reference. En Theories, Technology, Instrumentalities of Color. Anthropological and Historiographic Perspectives, B. Saunders \& J. Brakel, Eds., pp. 53-68. Lanham, MD: University of America Press.

Gage, J., 1993. Color and Culture: Practice and meaning from antiquity to abstraction. London: Thames \& Hudson.

GeLl, A., 1992. The technology of enchantment and the enchantment of technology. En Anthropology, Art and Aesthetics, J. Coote \& A. Shelton, Eds., pp. 40-66. Oxford: Clarendon Press. 1998. Art and Agency. An Anthropological Theory. Oxford: Clarendon Press.

Gombrich, E., 1999. El sentido del orden. Estudio sobre la psicología de las artes decorativas. Madrid: Debate.

Hardin, C. \& L. Maffi, 1997. Color Categories in Thought and Language. Cambridge: Cambridge University Press.

Ibarra Grasso, D. E., 1973. Prehistoria de Bolivia. La Paz: Los Amigos del Libro.

ITTEN, J., 1961. The Art of Color. New York: Van Nostrand Reinhold.

JONES, A. \& G. MACGREGOR, 2002. Colouring the past: The significance of colour in archaeological research. Oxford: Berg.

KANIZSA, G., 1986. Gramática de la visión: Percepción y pensamiento. Barcelona: Editorial Paidós.

KoffKA, K., 1973. Principios de la psicología de la forma. Buenos Aires: Editorial Paidós.

Krapovickas, P., 1987/1988. Nuevos fechados radiocarbónicos para el sector oriental de la Puna y la Quebrada de Humahuaca. Runa 17: 207-219.

KrapovicKas, P. \& S. AleKSANDrowicz, 1990. Breve visión de la cultura Yavi. Anales de arqueología y etnología 12-13: 83-127.

LATOuR, B., 2005 Reassembling the social: An introduction to actornetwork-theory. Oxford: Oxford University Press.

LuCY, J., 1997. The Linguistic of Color. En Color Categories in Thought and Language, Hardin \& Maffi, Eds., pp. 320-346. Cambridge: Cambridge University Press.

Mamaní, H., 1998. El paisaje arqueológico en el sector occidental de la cuenca de Pozuelos (Jujuy, Argentina). En Los desarrollos locales y sus territorios, M. B. Cremonte, Ed., pp. 257-284. San Salvador de Jujuy: Unju.

Miller, D., 2005. Materiality. Durham, NC: Duke University Press. Moles, A. \& L. JANISZEWSKI, 1992. Grafismo funcional. Enciclopedia del diseño. Barcelona: Ediciones CEAC.

Morphy, H., 1991. Ancestral connections: Art and an aboriginal system of knowledge. Chicago: Chicago University Press.

Munn, N., 1973. The Walbiri Iconography. Chicago: Chicago University Press.

Nielsen, A., 2007. Celebrando con los antepasados. Arqueología del espacio público en los Amarillos, Quebrada de Humabuaca, Jujuy, Argentina. Buenos Aires: Mallku.

Nielsen, A.; F. Ávila \& M. VÁzQuez, 2010. Notas sobre la arqueología de la cuenca media del río Grande de San Juan (1000-1450 DC). Ponencia presentada en el XVII Congreso Nacional de Arqueología Argentina, Mendoza.

Owoc, M., 2002. Munselling the Mound: The use of soil colour metaphor in British Bronze Age funerary ritual. En Colouring the past: The significance of colour in archaeological research, A. Jones \& G. MacGregor, Eds., pp. 127-140. Oxford: Berg.

Rafrino, R. A., 1988. Poblaciones indígenas de Argentina. Buenos Aires: Tea.

ReNDON, P., 2008. El conjunto cerámico meridional: Singularidades y variabilidad de la cerámica local de la microrregión del Saire relacionada a los estilos cerámicos Tarija, Yavi y Chicha. Arqueología de las tierras altas, valles interandinos y tierras bajas de Bolivia. Ponencia presentada en el I Congreso de Arqueología de Bolivia, La Paz.

Saunders, B., 2002. Getting in touch with the world. En Theories, Technology, Instrumentalities of Color. Anthropological and Historiographic Perspectives, B. Saunders \& J. Brakel, Eds., pp. 91-104. Lanham, MD: University of America Press. 
SAUNDERS, B. \& J. van BraKel, 1998. Re-evaluating basic color terms Cultural Dynamics 1: 359-378.

Sсотт, R., 1950. Fundamentos del diseño. Buenos Aires: Editorial Víctor Lerú.

Stovel, E., 2002. The importance of being atacameño: Political identity and mortuary ceramics in northern Chile. Tesis de doctorado en filosofía y antropología, Graduate School of Binghamton University, State University of New York.

TARragó, M., 1989. Contribuciones al conocimiento arqueológico de los oasis de San Pedro de Atacama en relación con los otros pueblos puneños, en especial, el sector septentrional del Valle
Calchaquí. Tesis de doctorado, Facultad de Humanidades, Universidad Nacional de Rosario.

TOREN, C., 1993. Making history: The significance of childhood cognition for a comparative anthropology of mind. Man 28: 461-478.

Turner, V., 1980. La selva de los símbolos. Buenos Aires: Siglo XXI

WierzBickA, A., 1990. The meaning of color terms: semantics, culture and cognition. Cognitive Linguistics 1 (1): 99-150.

Wong, W., 1979. Fundamentos del diseño bi y tridimensional. Barcelona: Editorial Gustavo Gili.

Young, D., 2006. The color of the things. En Handbook of Material Culture, C. Tilley, W, Keane, S. Küchler, M. Rowlands, P. Spyer, Eds., pp. 173-185. London: Sage Publications. 
